\title{
CORRECTION
}

\section{Correction: CSN6-TRIM21 axis instigates cancer stemness during tumorigenesis}

Baifu Qin, Shaomin Zou, Kai Li, Huashe Wang, Wenxia Wei, Boyu Zhang, Lishi Xiao, Hyun Ho Choi, Qin Tang, Dandan Huang, Qingxin Liu, Qihao Pan, Manqi Meng, Lekun Fang and Mong-Hong Lee

British Journal of Cancer (2020) 123:1042-1044; https://doi.org/10.1038/s41416-020-0977-5

Correction to: British Journal of Cancer (2020) 122, 1673-1685; https://doi.org/10.1038/s41416-020-0779-9, published online 30 March 2020

The original version of this article contained an error in Fig. 1f. The caption incorrectly listed 'Csn6 high and Aldh1a1 low $(n=59)$ ' as 'Csn6 low and Aldh1a1 low $(n=59)$ ', and 'Csn6 high and Aldh1a1 high $(n=120)$ ' as 'Csn6 low and Aldh1a1 high $(n=120)$ '. This correction has been made to the caption and a corrected version of the Figure is below. 


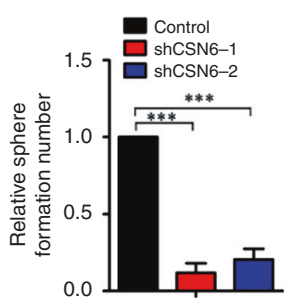

DLD1

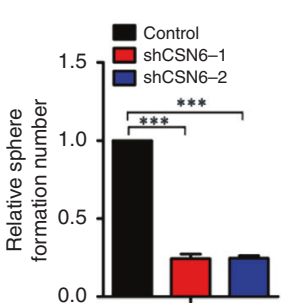

HCT116

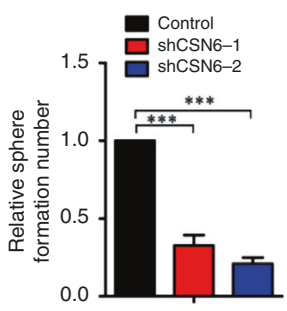

HCT-8

b

\begin{tabular}{|c|c|c|c|c|c|c|c|c|}
\hline & \multicolumn{4}{|c|}{ Sphere-containing wells/total wells } & \multirow{3}{*}{\multicolumn{2}{|c|}{ Sphere-initiating cell frequency }} & \multirow{3}{*}{$\begin{array}{c}\text { Fold } \\
\text { reduction }\end{array}$} & \multirow{3}{*}{ Probabilit } \\
\hline & \multirow[b]{2}{*}{100} & \multicolumn{2}{|c|}{ Cells per well } & \multirow[b]{2}{*}{2} & & & & \\
\hline & & 50 & 10 & & & & & \\
\hline Control & $32 / 32$ & $32 / 32$ & $43 / 48$ & $19 / 60$ & $1 / 5.26$ & $(1 / 6.77-1 / 4.12)$ & & \\
\hline ShCSN6-1 & $32 / 32$ & $30 / 32$ & $13 / 48$ & $3 / 60$ & $1 / 24.06$ & $(1 / 31.65-1 / 18.32)$ & 4.6 & *** \\
\hline ShCSN6-2 & $32 / 32$ & $25 / 32$ & $12 / 48$ & $2 / 60$ & $1 / 31.65$ & $(1 / 41.45-1 / 24.2)$ & 6.0 & 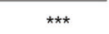 \\
\hline
\end{tabular}
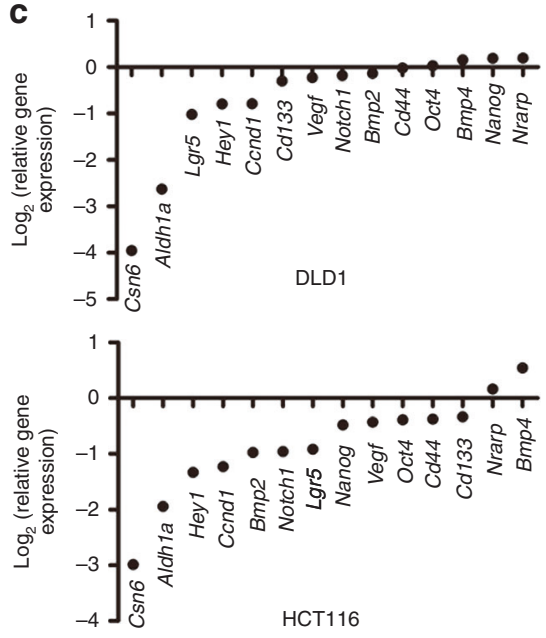

d
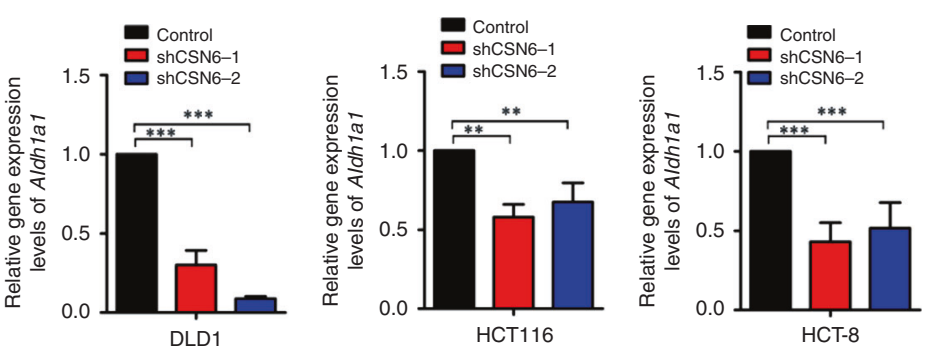

HCT-8
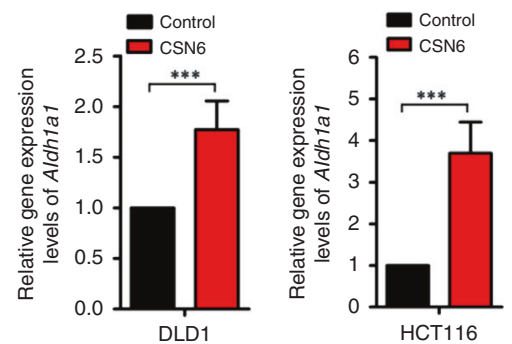

e

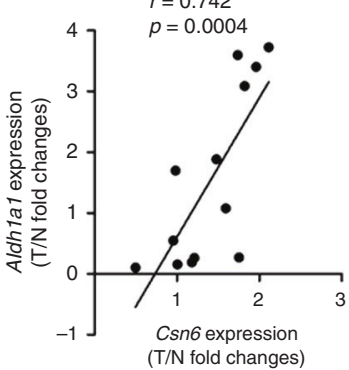

g

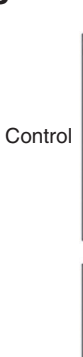

f GSE39582

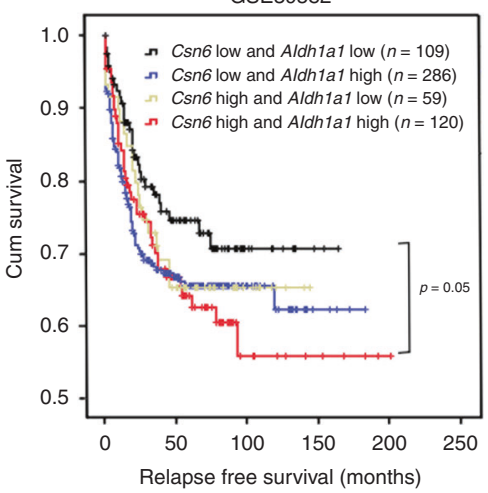

Day 0

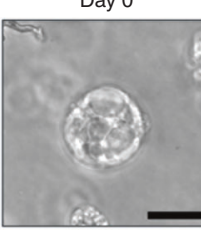

Day 3

Day 6

Day 9
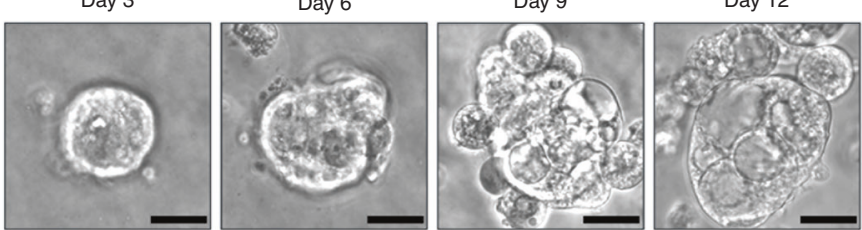

shCSN6
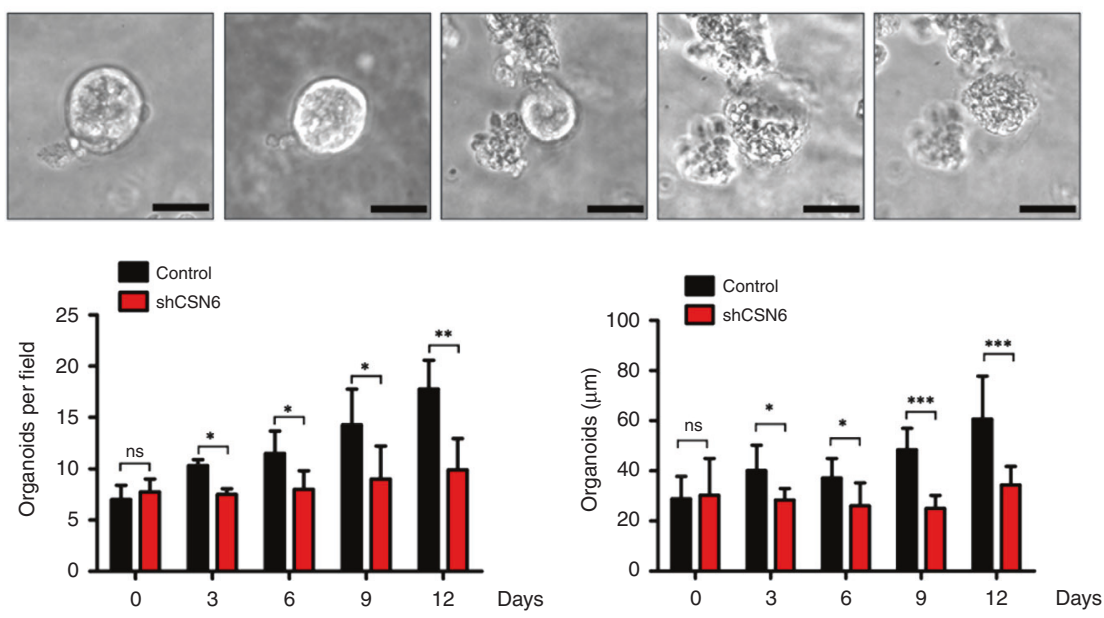
1044

Fig. 1 CSN6 is required for sphere formation and initiates stemness through ALDH1A1. a Sphere-formation assay of DLD-1, HCT116 and HCT-8 cells carrying scrambled or CSN6-specific shRNA. b DLD-1 cells carrying scrambled or CSN6-specific shRNA were dissociated into a single-cell suspension, seeded in 96-well plates with an ultra-low attachment surface at a density of 2, 10,50 or 100 cells per well and cultured for 12 days. The frequency of sphere-initiating cells was estimated using the ELDA software. c Quantitative RT-PCR analysis was performed to measure the mRNA levels of stem cell markers (Aldh1a1, Lgr5, Cd133 and Cd44), embryonic stem cell components (Nanog and Oct4), WNT pathway components (Vegf and Ccnd1), Notch pathway components (Notch1, Hey1 and Nrarp) and BMP family genes (Bmp2 and Bmp4) in DLD1 cells and HCT116 cells carrying scrambled or CSN6-specific shRNA. d Quantitative RT-PCR analysis was performed to measure the mRNA levels of Aldh1a1 in DLD-1, HCT116 and HCT-8 cells with CSN6 knockdown or CSN6 overexpression. e Quantitative RT-PCR analysis was performed to measure the mRNA levels of colorectal cancer and adjacent colorectal tissues. The levels of Csn6 were positively correlated with the expression of Aldh1a1 at mRNA levels in 13 pairs of human colorectal carcinomas (T) with matched normal tissues (N). $f$ Kaplan-Meier survival curves of relapse-free survival time based on Csn6 and Aldh1a1 expression in CRC tissues. ${ }^{*} P<0.05,{ }^{*} P<0.01$ and ${ }^{* * *} P<0.001$. g Knockdown of CSN6 affected patient-derived tumour organoid (tumour PDO) growth. The morphology of the organoids is shown. The number of organoids growing to a size of $>25 \mu \mathrm{m}$ was calculated. Scale bars, $25 \mu \mathrm{m}$. 